\title{
Energy Efficient Wireless Sensor Networks based on QoS Enhanced Base Station controlled Dynamic Clustering Protocol
}

\author{
M.Sheik Dawood \\ Associate professor \\ Sethu institute of technology \\ Pulloor, India
}

\author{
S.Sadasivam \\ Associate professor \\ Sethu institute of technology \\ Pulloor, India
}

\author{
G.Athisha \\ Professor \\ PSNA College of Engg. \& tech \\ Dindigul, India
}

\begin{abstract}
Recent development in Micro Electro Mechanical System (MEMS) technology leads to the development of low cost and low power utilizing wireless micro sensor nodes. Wireless sensor network is a power consuming scheme, because nodes executes on restricted power batteries which reduces its lifetime. Extending the network lifetime is based on the better managing of sensing node energy resource. Energy efficient routing protocol is very essential technique in wireless sensor networks because sensor nodes are extremely energy based. As a result, several researchers have provided different routing protocol for sensor networks, particularly routing protocols depending on clusters protocols. This is because the usage of cluster based routing has several merits like minimized control messages, re-usability of bandwidth and enhanced power control. Different cluster based routing protocol is proposed by many researchers for the purpose of reducing the consumption energy in wireless sensor networks. Those technique reduces the energy consumption but with several disadvantages like lack of QoS, inefficient transmission, etc., To overcome those problems, modified QoS enhanced base station controlled dynamic clustering protocol for wireless sensor networks is proposed in this paper. Simulation results show the better energy consumption is achieved by the proposed protocol when compared to the conventional techniques.
\end{abstract}

\section{Index terms}

Wireless Sensor Network, Clustering protocol, QoS

\section{Keywords}

Clustering, Energy efficiency, Lifetime.

\section{INTRODUCTION}

Wireless Sensor Network[3] is an unique variety of Ad hoc networks that turn out to be attractive field for researchers. Routing protocol is the major thing to considering networks where available resources are restricted. Wireless Sensor Network technique's development in the computation capability needs the sensor nodes to be used more to deal with more complex function. Every sensor is usually restricted in their energy level.

The important difficulty in the organization of sensor networks is energy efficiency [11]. This requirement for energy efficiency occurs since the sensor node battery capability is strictly restricted and battery replacement is not practical. The sensor node battery restriction reduces the lifetime of the network. The lifetime of every single node differs according to the requirements positioned on its battery. Therefore, a main factor in the construction of sensor networks is their robustness to handle the falling life time of every sensor nodes. Different network architectures and routing protocols to reduce energy consumption [14] and to expand sensor network lifetime has been analyzed. After analyzing those techniques, network construction dependent on clustering are regarded as the efficient technique in when energy consumption $[7,8]$ is considered. In this technique, sensor nodes are structured into clusters, and single sensor node in every cluster is chosen as the Cluster Head $(\mathrm{CH})$ which is then responsible to act as a transfer point. In addition, every cluster head generates a transmission plan for the sensor nodes presented in the cluster. This plan permits the radio components of every non-cluster hear-node to be powered down apart from during permitted transmit times.

There are several clustering based protocols [9] proposed by many authors. All those methods have their own advantages and disadvantages. For reducing the energy consumption, the modified QoS enhanced base station controlled dynamic clustering protocol for wireless sensor network is proposed in this paper which can be able to reduce the energy consumption effectively. Low Energy Adaptive Clustering Hierarchy (LEACH) technique is used in the clustering phase of the proposed technique.

\section{RELATED WORKS}

In Wireless sensor networks where the source nodes are close to one another and generate a lot of sensed data, traffic with redundancy, transmitting all sensed data by individual nodes not only wastes the scarce wireless bandwidth, but also consumes a lot of battery energy. In this paper, Babak et al., [1] introduced an algorithm, which make better use of energy and bandwidth that are two restrictions in wireless sensor networks. In the algorithm, a mobile agent is used to cluster the network and also create the tour to obtain collected data from each cluster-head and deliver back to the sink node. With appropriate parameters set, simulation shows that proposed algorithm exhibits better performance than original direct diffusion in terms of energy consumption [15].

The principles energy optimization with multi-level clustering algorithm for wireless sensor networks is presented by Rasid 
et al. [2, 4].In order to minimize the amount of data transmission from the energy efficiency perspective, clustering approach is featured by routing algorithm used in the wireless. But, the clustering approach results in high energy consumption at the cluster head node. Dynamic clustering is an approach used to resolve such a problem by distributing energy consumption through the re-selection of the cluster head node. Still, dynamic clustering alters the cluster structure every time the cluster head node is reselected, which causes energy consumption. In other words, the dynamic clustering techniques observed in previous studies involve the repetitive processes of cluster head node selection. This takes a high amount of energy during the setup process of cluster generation. To resolve the energy consumption problem associated with the repetitive set-up, this paper proposes the round-robin cluster header $(\mathrm{RRCH})$ method that fixes the cluster and selects the head node in a round-robin method The $\mathrm{RRCH}$ approach is an energyefficient method that realizes consistent and balanced energy consumption in each node of a generated cluster to prevent repetitious set-up processes as in the LEACH method. The propriety of the proposed approach is substantiated with a simulation experiment.

Clustering [12, 13] is one of the key approaches used in wireless sensor networks to save energy. The clustering is affected by the transmission range and in turn affects the energy consumption in the network. Bolian et al., [5] established an energy consumption approach for clustered wireless sensor networks and solve the optimal transmission range problem. Using this approach, the total energy consumption can be estimated beforehand based on the traffic pattern, energy model, and network deployment parameters. This technique presents an insight into the energy consumption behavior in clustered wireless sensor networks and the relationship among major factors. The optimal transmission range for energy consumption is a function of the traffic load and the node density, but the effect of node density is very limited.

The reduction of energy consumption [10] in wireless sensor networks is challenging issue, and grid-based clustering and routing schemes is playing a vital role due to their simplicity and feasibility. Thus, determining the optimal grid size in order to minimize energy consumption and prolong network lifetime becomes an important problem during the network planning and dimensioning phase. The previous researches have used the average distances within a grid and between neighbor grids to calculate the average energy consumption, which largely underestimates the real value. In this paper, Yanyan et al., [6] proposed, analyzed and evaluated the energy consumption models in wireless sensor networks with probabilistic distance distributions. These techniques have been validated by numerical and simulation results, which shows that they can be used to optimize grid size and minimize energy consumption accurately. The author also uses these approaches to study variable-size grids, which can further improve the energy efficiency by balancing the relayed traffic in wireless sensor networks.

\section{Methodology}

\subsection{Wireless Sensor Network Model}

The network model considered in this paper is as follows:

i. The wireless sensor network is considered that contains $M$ sensor nodes in scattered manner in a sensor field. The operation situation is illustrated in figure 1 , here the sensor field is a square area of side $L$ at a distance $d_{B S}$ from a lone fixed base-station.

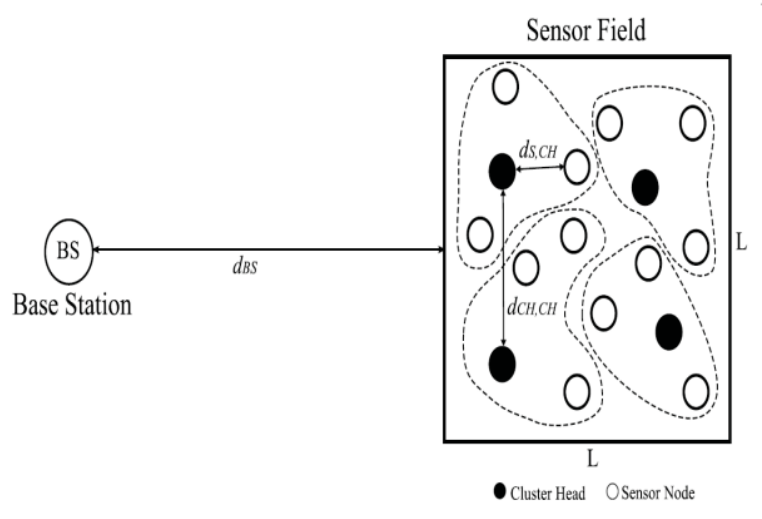

Figure 1. Example wireless sensor network model.

ii. The $M$ sensor nodes are provided with limitations in battery power, processing power and memory space.

iii. The $M$ sensor nodes are fixed and are grouped physically into clusters. The nodes in a cluster may possibly carry out any of the two functions: cluster head or sensing. Every cluster head carry out functions like scheduling of intracluster and intercluster communications, data aggregation and data forwarding to the base station with the help of multi-hop routing. Conversely, a cluster head node cannot carry out the sensing process. The purpose of cluster head is rotated between the non-sensing nodes in a cluster. Alternatively, a sensing node will be dynamically sensing the situation or in the inactive mode if it is not sensing. The nodes to be used for sensing tasks are decided by the base station.

iv. The data sensed by the sensing nodes in a cluster are broadcasted straightly to their cluster head that afterwards aggregates and/or pass the data to another cluster head which will direct it to the base station. Different from the sensor nodes, the base station is not with the restricted resources. Thus the communication from the base station to the sensor nodes can be performed straight forwardly.

$\mathrm{v}$. The base station has information of the position of every node in the network that is situated within the sensor field.

QoS Enhanced Base Station Controlled Dynamic Clustering Protocol (QBCDCP)assume homogeneity in sensor node capability and constraints due to its simplicity of its deployment and investigation, and for reliability with most of the offered work in routing for wireless sensor network. But there are several applications that would advantage from QoS 
routing for networks with homogeneous nodes, and methods presented here to realize satisfactory QoS and lesser consumption of energy may also be appropriate to the construction of protocol handling with heterogeneous networks. The supposition of immobile nodes is based on the detail that, for several applications, sensor nodes will be fixed at certain position after deployment, because the energy necessary to offer mobility to such nodes is unaffordable.

\subsection{QoS Enhanced Base Station Controlled Dynamic Clustering Protocol}

QBCDCP is an improved technique of BCDCP with the recently added functionality of QoS based route selection. QoS is maintained in QBCDCP by including delay and bandwidth detail in route selection. Also the energy necessary for transmission is considered here. The functional components of QBCDCP and the associations among them are depicted in figure 2. Every data round, specified by a fixed time interval $t_{D R}$, the base station groups the sensor nodes into balanced clusters with the help of LEACH.

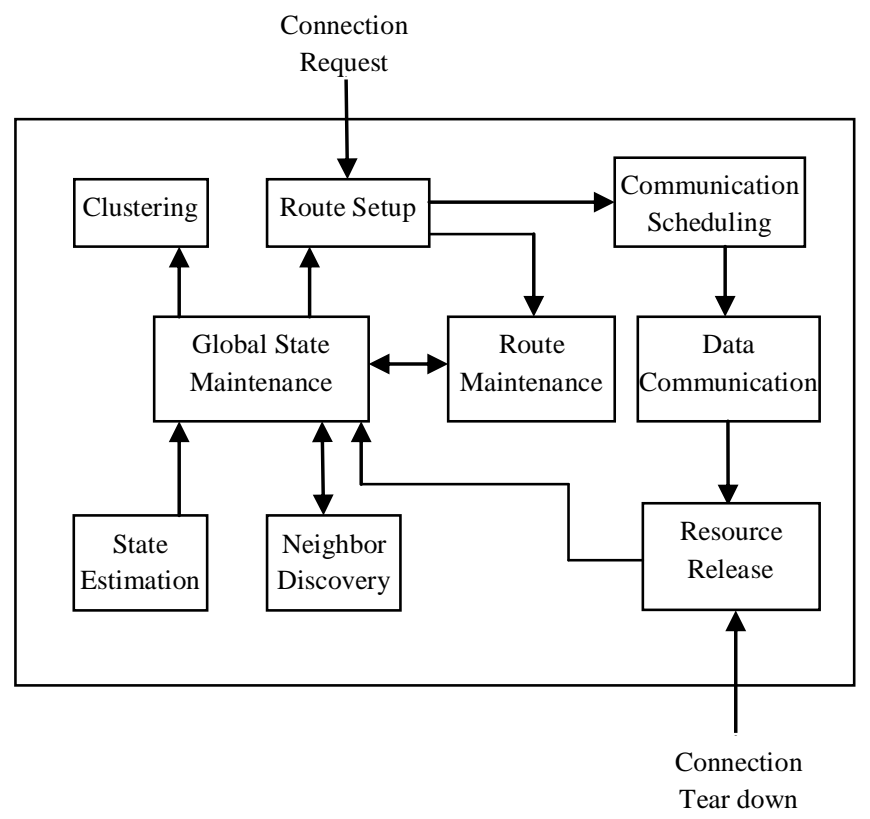

Figure 2. QoS Enhanced Base Station Controlled Dynamic Clustering Protocol

\subsection{Clustering Process}

Low Energy Adaptive Clustering Hierarchy (LEACH) cluster heads are stochastically chosen. For selecting cluster-heads every node $n$ finds a random number between 0 and 1 . If the number is below a threshold $\mathrm{T}(\mathrm{n})$, the node is decided as a cluster-head for the present round. The threshold is determined as follows:

$$
\begin{array}{cr}
T(n)=\frac{P}{1-P \times\left(\operatorname{rmod} \frac{1}{p}\right)} \forall n \in G \\
T(n)=0 \quad \forall n \notin G
\end{array}
$$

Where $\mathrm{P}$ is the cluster-head probability, $\mathrm{r}$ is the number of the present round and $\mathrm{G}$ is the set of nodes that is not the clusterheads in the final 1/P rounds. This algorithm guarantees that each node turn out to be a cluster-head accurately once inside $1 / \mathrm{P}$ rounds.

Considering the single round of $\mathrm{LEACH}$, it is clear that a stochastic cluster-head choosing will not automatically direct to lesser energy consumption during data transmit for provided nodes. Every cluster-heads can be situated close to the edges of the network or nearby nodes can turn into cluster heads. In this situation several nodes have to link long distances to attain a cluster-head. On the other hand, considering the two or more rounds it could be implicit that choosing appropriate cluster-heads outcome in an adverse cluster-head selection in upcoming rounds because LEACH attempts to dispense energy consumption between every nodes.

In the worst-case-situation cluster-heads are chosen unfavorably close to the edges, in round 0 on the right hand side and in round 1 on the left hand side of the network. In the best-case-situation cluster-heads are not scattered favorably along the network, but, better than in the worst case situation. The gathered transmission energy of every node over both rounds currently report for $90.5 \mathrm{~nJ} / \mathrm{bit}$. Next, a deterministic cluster-head selection algorithm with reduced energy consumption is presented.

The initial technique enhancing the life span of a LEACH network is the addition to the remaining energy level existing in every node. It can be accomplished by decreasing the threshold T (n), comparative to the node's left out energy. Thus, $\mathrm{T}(\mathrm{n})$ is multiplied with an aspect representing the remaining energy level of a node:

$$
T(n)_{n e w}=\frac{P}{1-P\left(\operatorname{rmod} \frac{1}{P}\right)} \frac{E_{n \_ \text {current }}}{E_{n \_ \text {max }}}
$$

Where $E_{n_{-} \text {current }}$ indicates the current energy and $E_{n \_ \text {max }}$ indicates the initial energy of the node.

However, an alteration of the threshold equation by the left out energy has an important demerit i.e., after some number of rounds the network is jammed, even though there are some nodes existing with sufficient energy to broadcasting formation to the base station. This is because the cluster-head threshold is very low, since the lasting nodes have a lesser energy level.

A probable answer for this difficulty is an additional alteration of the threshold equation. It is extended by a factor that enlarges the threshold for some node that is not cluster head for the previous $1 / \mathrm{P}$ rounds: 


$$
\begin{aligned}
& T(n)_{n e w}=\frac{P}{1-P\left(\operatorname{rmod} \frac{1}{P}\right)}\left[\frac{E_{n_{-} \text {current }}}{E_{n_{-} \max }}\right. \\
& \left.+\left(r_{s} \operatorname{div} \frac{1}{P}\right)\left(1-\frac{E_{n_{-} \text {current }}}{E_{n \_ \text {max }}}\right)\right]
\end{aligned}
$$

Where $r_{s}$ represents the number of successive rounds in that a node has not been cluster-head. When $r_{s}$ attains the value 1/P the threshold $\mathrm{T}(\mathrm{n})$ new is changed to the value it had previous to the addition of the left out energy into the threshold equation. Therefore, the possibility of node $\mathrm{n}$ to turn into cluster-head boosted since it has a higher threshold. A probable barrier of the network is resolved. In addition, $r_{\mathrm{s}}$ is reset to 0 after a node turns into cluster head. Therefore, it is guaranteed that data is broadcasted to the base station until the nodes are alive.

The clustered character of the network and the necessity of scheduled broadcastings by sensor nodes, the medium access control protocol chosen for QBCDCP is the time division multiple access. The time division multiple access structure for $\mathrm{QBCDCP}$ is regarded in that the frame length is considered with length $T$ time units, partitioned into $v$ time slots, one of which is kept for control and the other slots are segmented for receiving and sending the data messages. The control slot is helpful in sending and receiving of control messages associated to clustering and routing information, state updates data requests and acknowledgments, irregularity finding and neighbor detection.

For permitting the concurrent transmissions in adjacent clusters and, therefore, decrease inter-cluster interference, every cluster is allocated with a specific spreading code supposed to be orthogonal. When a link is demanded, the base station sets up a QoS-based route Q between the cluster head where the connection is created by its own. The route $\mathrm{Q}$ is indicated as a series of pairs of $\mathrm{CH}$ nodes: $\mathrm{Q}=\{(0,1),(1,2)$. . $(H, B S)\}$ where every pair determines the connection that corresponds to route $\mathrm{Q}$ and $H$ is the hop length between cluster head 0 and the base station. Every directed connection $(i, j)$, where node $i(j)$ is the originating end of the connection, ( $i \in\{0,1,2, \ldots\},, j \in\{1,2, \ldots, B S\})$ is allocated a metric described in terms of its bandwidth, delay and transmission energy. The transmission energy related with link $(i, j),(d)$, is the power amplifier energy of node $i$, that is a function of $d_{i j}$, the distance between cluster head nodes $i$ and $j$, and the implicit propagation method. The delay corresponding with connection $(i, j)$ is indicated by $D_{j}$, the average delay obtained by packets traversing channel head node $j$, and the existing bandwidth corresponding to the link $(i, j), B W_{i j}$, is the least of the existing bandwidths at nodes $i$ and $j$. When routing is carried on, a cost metric $c_{i j}$ that contains the linear combination of link $(i, j)$ 's transmission energy and delay is created, for all the links $(i, j)$ in the network:

$$
C_{i j}=\alpha E_{a m p}\left(d_{i j}\right)+(1-\alpha) D_{j}, \quad \forall \operatorname{link}(i, j)
$$

Where $\alpha$ indicates is a configurable parameter that is kept to any range between $[0,1]$ and allocates corresponding weights to delay and transmission energy metrics for route selection. The function $\left(d_{i j}\right)=\varepsilon_{p} d_{i j}{ }^{\nu}$, where, if $d_{i j} \leq d_{0}, \gamma=2$ and $\varepsilon_{p}=$ $\varepsilon_{F S}$, will represent free space power amplification factor. On the other hand if $d_{i j}>d_{0}, \gamma=4$ and $\varepsilon_{p}=\varepsilon_{T R}$, will represent two-ray amplification factor. The threshold distance $d_{0}=$ $\sqrt{\varepsilon_{F S} / \varepsilon_{T R}}$. Next, the QoS routing difficulty therefore consists of determining a path $\mathrm{Q}$ for a link that decreasesCpath, the total of the costs $c i j$ of the connections along the path, while assuring both the bandwidth and delay objectives for the connection, represented by $B W_{\text {req }}$ and $D_{\text {req }}$, correspondingly[16]. Mathematically,

$$
\arg \min _{Q}\left(C_{\text {path }}=\sum_{\forall(i, j) \in Q} c_{i j}\right)
$$

$$
\begin{gathered}
B W_{\text {path }}=\min \left(B W_{i j}\right) \geq B W_{r e q} \\
D=f\left(\sum_{\forall(i, j) \in Q} D_{j}\right) \leq D_{e q}
\end{gathered}
$$

Where $B W_{\text {path }}$ represents the assigned bandwidth for the route and $D$ is the deliberated as end-to-end delay for an event measured from the moment of its sense until its reception by the base station. Clearly $D$ is a function of the total of delays of the connections along the chosen path $\mathrm{Q}$. When the route selection procedure is carried on, the base station selects cluster heads that will produce the route with the help of left out energy, so that the $\mathrm{CHs}$ have sufficient energy to communicate with the links. As links are arranged, the bandwidth metric of every cluster head is updated, so cluster heads only consider those links they can function. When a QoS route has been chosen, the route is then promoted by the BS to the cluster head nodes along the path, demanding the nodes to schedule the connection. This procedure is known as slot assignment or communication scheduling. The slot assignment is carried on locally by the involved cluster head nodes, by the help of slot negotiation process. The slot allocation is flourishing only when the demandeds slots are free at both nodes $i$ and $j$, or else, an error message is sent back to the BS to point out that the scheduling of the connection is failed. For the purpose of simplifying the slot assignment process, intercluster communication is scheduled first, and then intracluster communication is performed.

\section{EXPERIMENTAL RESULTS}

The network considered for the simulation consists of 200 nodes distributed randomly across an area of 100x100 meters. Every node is prepared with an energy source whose total amount of energy accounts for $1 \mathrm{~J}$ at the starting of the simulation. Each node transmits a 200-bit message per round to its actual cluster head. 


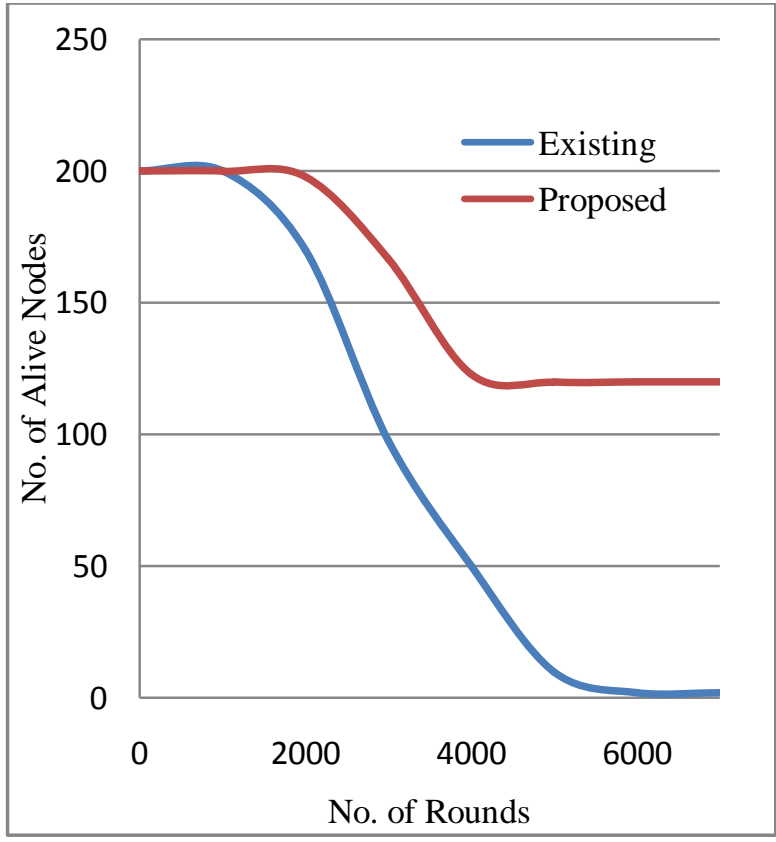

Figure 3. Number of Alive Nodes per Round

Figure 3 shows the simulation result comparison for the proposed method and the existing method. From the graph, it can be observed that initially there are 200 nodes in the network before starting execution. After 1000 rounds of execution, the number of alive nodes for the existing and proposed method are same i.e., 200. When 2000 executions are completed, the number of alive nodes for the existing method is 170 , whereas, 198 nodes remains by using the proposed technique. After 3000 executions are completed, 167 nodes remaining by using the proposed technique and only 98 nodes are remaining by using the existing method. When 4000 executions are completed, the number of alive nodes for the existing method is 50, whereas, 120 nodes remains by using the proposed technique. When 6000, executions are completed, all the nodes in the network are dead, whereas, still 120 nodes remains by using the proposed approach for routing. This clearly indicates the less energy consumption by using the proposed protocol for routing when compared to the existing methods.

\section{Conclusion}

Wireless Sensor Networks are a type of Adhoc networks that leads to one of the very attractive field for researchers. Routing protocol is the most significant issue for networks when resources are restricted. The growth of WSN with high computation capability needed the sensor nodes must be designed in order to deal with very complex functions. Every sensor is usually restricted in their energy level, processing power and sensing capability. It is required to make use of the available energy efficiently. To achieve this, modified QoS enhanced base station controlled dynamic clustering protocol for wireless sensor networks is proposed in this paper. The simulation result shows that the proposed technique results in

[11] C. Schurgers and M. B. Srivastava, "Energy Efficient Routing in Wireless Sensor Networks," in proc. IEEE more number of live nodes when compared to the proposed technique. This clearly represents the lesser energy consumption by using the proposed clustering based routing protocol when compared to the existing protocols for wireless sensor network.

\section{REFERENCES}

[1] BabakNikmard and Salman Taherizadeh, "Using mobile agent in clustering method for energy consumption in wireless sensor network", International Conference on Computer and Communication Technology (ICCCT), pp.153-158, 2010.

[2] Rasid M.F.A., Abdullah, R.S.A., Ghazvini M.H.F., and Vahabi M., "Principles Energy Optimization with Multilevel Clustering Algorithm for Wireless Sensor Networks", IFIP International Conference on Wireless and Optical Communications Networks, 2007. WOCN '07, pp.1-5, 2007.

[3] Do-hyun Nam and hong-ki min, "An Energy-Efficient Clustering Using a Round-Robin Method in a Wireless Sensor Network", 5th ACIS International Conference on Software Engineering Research, Management \& Applications (SERA), pp.54-60, 2007.

[4] Ghazvini M.H.F., Vahabi M., Rasid M.F.A., and Abdullah R.S.A., "Principles Optimizing energy consumption in hierarchical clustering algorithm for wireless sensor networks", IEEE International Conference on Telecommunications and Malaysia International Conference on Communications, ICTMICC, pp.550-55, 2007.

[5] Bolian Yin, Hongchi Shi and Yi Shang, "Analysis of Energy Consumption in Clustered Wireless Sensor Networks", 2nd International Symposium on Wireless Pervasive Computing, ISWPC '07, 2007.

[6] YanyanZhuang, Jianping Pan and Lin Cai, "Minimizing Energy Consumption with Probabilistic Distance Models in Wireless Sensor Networks", NFOCOM, 2010 Proceedings IEEE, pp.1-9, 2010.

[7] Malik A.S., Jingming Kuang, Jiakang Liu and Wang Chong, "Energy Consumption \& Lifetime Analysis in Cluster-Based Wireless Sensor Networks for Periodic Monitoring Applications", International Conference on Networks Security, Wireless Communications and Trusted Computing, 2009. NSWCTC '09, Vol.1, pp.657661, 2009.

[8] C. B. Margi, V. Petkov, V. Obraczka, and R. Manduchi, "Characterizing energy consumption in a visual sensor network testbed." Available online: http://www.cse.ucsc.edu/manduchi/papers/stargateenergy .pdf

[9] S. D. Muruganathan, D. C. F. Ma, R. I. Bhasin, and A. O. Fapojuwo, "A centralized energy-efficient routing protocol for wireless sensor networks," IEEE Commun. Mag., Vol. 43, No. 3, pp. S8-S13, 2005.

[10] Ahmed I., MugenPeng and Wenbo Wang, "Uniform Energy Consumption through Adaptive Optimal Selection of Cooperative MIMO Schemes in Wireless Sensor Networks", Vehicular Technology Conference, VTC Spring, pp.198-202, 2008.

Military Communications Conf. Vol. 1, pp. 357-361. 2001. 
[12] C. F. Li, M. Ye, G. Chen, and J. Wu, "An EnergyEfficient Unequal Clustering Mechanism for Wireless Sensor Networks," IEEE International Conf. Mobile Adhoc and Sensor Systems, pp. 8, Nov. 2005.

[13] YanyanZhuang, Jianping Pan and Guoxing $\mathrm{Wu}$, "Energy-Optimal Grid-Based Clustering in Wireless Microsensor Networks", 29th IEEE International Conference on Distributed Computing Systems Workshops, 2009. ICDCS Workshops '09, pp.96-102, 2009.

[14] Gagarin A., Hussain S., and Yang, L.T., "Distributed Search for Balanced Energy Consumption Spanning Trees in Wireless Sensor Networks", International
Conference on Advanced Information Networking and Applications Workshops, WAINA '09, pp.1037-1042, 2009.

[15] Ali K., Neogy S., and Das P.K., "Optimal Energy-Based Clustering with GPS-Enabled Sensor Nodes", Fourth International Conference on Sensor Technologies and Applications (SENSORCOMM), pp.13-18, 2010.

[16] Abraham O. Fapojuwo, Senior Member, IEEE, and Alejandra Cano-TinocoEnergy Consumption and Message Delay Analysis of QoS Enhanced Base Station Controlled Dynamic Clustering Protocol for Wireless Sensor Networks, IEEE transactions on wireless communications, vol. 8, no. 10, October 2009 